\title{
RESEARCH
}

\section{On the least common multiple of random $q$-integers}

\section{Carlo Sanna* (1)}

\section{${ }^{*}$ Correspondence:}

carlo.sanna.dev@gmail.com Department of Mathematical Sciences, Politecnico di Torino, Corso Duca degli Abruzzi 24,

10129 Torino, Italy

C. Sanna is a member of GNSAGA of INdAM and of CrypTO, the group of Cryptography and Number Theory of Politecnico di Torino
Springer

\begin{abstract}
For every positive integer $n$ and for every $\alpha \in[0,1]$, let $\mathcal{B}(n, \alpha)$ denote the probabilistic model in which a random set $\mathcal{A} \subseteq\{1, \ldots, n\}$ is constructed by picking independently each element of $\{1, \ldots, n\}$ with probability $\alpha$. Cilleruelo, Rué, Šarka, and Zumalacárregui proved an almost sure asymptotic formula for the logarithm of the least common multiple of the elements of $\mathcal{A}$. Let $q$ be an indeterminate and let $[k]_{q}:=1+q+q^{2}+\cdots$ $+q^{k-1} \in \mathbb{Z}[q]$ be the $q$-analog of the positive integer $k$. We determine the expected value and the variance of $X:=\operatorname{deg} \operatorname{lcm}\left([\mathcal{A}]_{q}\right)$, where $[\mathcal{A}]_{q}:=\left\{[k]_{q}: k \in \mathcal{A}\right\}$. Then we prove an almost sure asymptotic formula for $X$, which is a $q$-analog of the result of Cilleruelo et al.

Keywords: Asymptotic formula, Least common multiple, $q$-analog, Random set

Mathematics Subject Classification: Primary: 11N37, Secondary: 11B99
\end{abstract}

\section{Introduction}

A nice consequence of the Prime Number Theorem is the asymptotic formula

$$
\log \operatorname{lcm}(1,2, \ldots, n) \sim n, \quad \text { as } n \rightarrow+\infty,
$$

where $\mathrm{lcm}$ denotes the least common multiple. Indeed, precise estimates for $\log \mathrm{lcm}(1, \ldots$, $n$ ) are equivalent to the Prime Number Theorem with an error term. Thus, a natural generalization is to study estimates for $L_{f}(n):=\log \operatorname{lcm}(f(1), \ldots, f(n))$, where $f$ is a wellbehaved function, for instance, a polynomial with integer coefficients. (We ignore terms equal to 0 in the $\operatorname{lcm}$ and we set $\operatorname{lcm} \varnothing:=1$.) When $f \in \mathbb{Z}[x]$ is a linear polynomial, the product of linear polynomials, or an irreducible quadratic polynomial, asymptotic formulas for $L_{f}(n)$ were proved by Bateman et al. [3], Hong et al. [10], and Cilleruelo [6], respectively. In particular, for $f(x)=x^{2}+1$, Rué et al. [15] determined a precise error term for the asymptotic formula. When $f$ is an irreducible polynomial of degree $d \geq 3$, Cilleruelo [6] conjectured that $L_{f}(n) \sim(d-1) n \log n$, as $n \rightarrow+\infty$, but this is still an open problem. However, bounds for $L_{f}(n)$ were proved by Maynard and Rudnick [13], and Sah [16]. Moreover, Rudnick and Zehavi [14] studied the growth of $L_{f}(n)$ along a shifted family of polynomials.

Another direction of research consists in considering the least common multiple of random sets of positive integers. For every positive integer $n$ and every $\alpha \in[0,1]$, let

(c) The Author(s) 2021. This article is licensed under a Creative Commons Attribution 4.0 International License, which permits use, sharing, adaptation, distribution and reproduction in any medium or format, as long as you give appropriate credit to the original author(s) and the source, provide a link to the Creative Commons licence, and indicate if changes were made. The images or other third party material in this article are included in the article's Creative Commons licence, unless indicated otherwise in a credit line to the material. If material is not included in the article's Creative Commons licence and your intended use is not permitted by statutory regulation or exceeds the permitted use, you will need to obtain permission directly from the copyright holder. To view a copy of this licence, visit http://creativecommons.org/licenses/by/4.0/. 
$\mathcal{B}(n, \alpha)$ denote the probabilistic model in which a random set $\mathcal{A} \subseteq\{1, \ldots, n\}$ is constructed by picking independently each element of $\{1, \ldots, n\}$ with probability $\alpha$. Cilleruelo et al. [9] studied the least common multiple of the elements of $\mathcal{A}$ and proved the following result (see [1] for a more precise version, and $[4,5,7,8,12,17-19]$ for other results of a similar flavor).

Theorem 1.1 Let $\mathcal{A}$ be a random set in $\mathcal{B}(n, \alpha)$. Then, as $\alpha n \rightarrow+\infty$, we have

$$
\log \operatorname{lcm}(\mathcal{A}) \sim \frac{\alpha \log (1 / \alpha)}{1-\alpha} \cdot n,
$$

with probability $1-o(1)$, where the factor involving $\alpha$ is meant to be equal to 1 for $\alpha=1$.

Remark 1.1 In the deterministic case $\alpha=1$, we have $\mathcal{A}=\{1, \ldots, n\}$ (surely) and Theorem 1.1 corresponds to (1).

Let $q$ be an indeterminate. The $q$-analog of a positive integer $k$ is defined by

$$
[k]_{q}:=1+q+q^{2}+\cdots+q^{k-1} \in \mathbb{Z}[q] .
$$

The $q$-analogs of many other mathematical objects (factorial, binomial coefficients, hypergeometric series, derivative, integral...) have been extensively studied, especially in Analysis and Combinatorics $[2,11]$. For every set $\mathcal{S}$ of positive integers, let $[\mathcal{S}]_{q}:=\left\{[k]_{q}: k \in\right.$ $\mathcal{S}\}$.

The aim of this paper is to study the least common multiple of the elements of $[\mathcal{A}]_{q}$ for a random set $\mathcal{A}$ in $\mathcal{B}(n, \alpha)$. Our main results are the following:

Theorem 1.2 Let $\mathcal{A}$ be a random set in $\mathcal{B}(n, \alpha)$ and put $X:=\operatorname{deg} \operatorname{lcm}\left([\mathcal{A}]_{q}\right)$. Then, for every integer $n \geq 2$ and every $\alpha \in[0,1]$, we have

$$
\mathbb{E}[X]=\frac{3}{\pi^{2}} \cdot \frac{\alpha \operatorname{Li}_{2}(1-\alpha)}{1-\alpha} \cdot n^{2}+O\left(\alpha n(\log n)^{2}\right),
$$

where $\operatorname{Li}_{2}(z):=\sum_{k=1}^{\infty} z^{k} / k^{2}$ is the dilogarithm and the factor involving $\alpha$ is meant to be equal to 1 when $\alpha=1$. In particular,

$$
\mathbb{E}[X] \sim \frac{3}{\pi^{2}} \cdot \frac{\alpha \operatorname{Li}_{2}(1-\alpha)}{1-\alpha} \cdot n^{2},
$$

as $n \rightarrow+\infty$, uniformly for $\alpha \in[0,1]$.

Theorem 1.3 Let $\mathcal{A}$ be a random set in $\mathcal{B}(n, \alpha)$ and put $X:=\operatorname{deg} \operatorname{lcm}\left([\mathcal{A}]_{q}\right)$. Then there exists a function $\mathrm{v}:(0,1) \rightarrow \mathbb{R}^{+}$such that, as $\alpha n /\left((\log n)^{3}(\log \log n)^{2}\right) \rightarrow+\infty$, we have

$$
\mathbb{V}[X]=(\mathrm{v}(\alpha)+o(1)) n^{3} .
$$

Moreover, the upper bound

$$
\mathbb{V}[X] \ll \alpha n^{3},
$$

holds for every positive integer $n$ and every $\alpha \in[0,1]$.

As a consequence of Theorems 1.2 and 1.3, we obtain the following $q$-analog of Theorem 1.1 . 
Theorem 1.4 Let $\mathcal{A}$ be a random set in $\mathcal{B}(n, \alpha)$. Then, as $\alpha n \rightarrow+\infty$, we have

$$
\operatorname{deg} \operatorname{lcm}\left([\mathcal{A}]_{q}\right) \sim \frac{3}{\pi^{2}} \cdot \frac{\alpha \operatorname{Li}_{2}(1-\alpha)}{1-\alpha} \cdot n^{2},
$$

with probability $1-o(1)$, where the factor involving $\alpha$ is meant to be equal to 1 for $\alpha=1$.

Remark 1.2 In the deterministic case $\alpha=1$, we have (see Lemma 4.1 below)

$$
\operatorname{deg} \operatorname{lcm}[\{1,2, \ldots, n\}]_{q}=\sum_{1<d \leq n} \varphi(d),
$$

and Theorem 1.4 corresponds to the well-known asymptotic formula $\sum_{d \leq n} \varphi(d) \sim \frac{3}{\pi^{2}} n^{2}$ (Lemma 3.3 below) for the sum of the first values of the Euler function $\varphi$.

Remark 1.3 In Theorem 1.4 the condition $\alpha n \rightarrow+\infty$ is necessary. Indeed, if $\alpha n \leq C$, for some constant $C>0$, then

$$
\mathbb{P}[\mathcal{A}=\varnothing]=(1-\alpha)^{n} \geq\left(1-\frac{C}{n}\right)^{n} \rightarrow \mathrm{e}^{C}
$$

as $n \rightarrow+\infty$, and so no (nontrivial) asymptotic formula for $\operatorname{deg} \operatorname{lcm}\left([\mathcal{A}]_{q}\right)$ can hold with probability $1-o(1)$.

We conclude this section with some possible questions for further research on this topic. Alsmeyer, Kabluchko, and Marynych [1, Corollary 1.5] proved that, for fixed $\alpha \in[0,1]$ and for a random set $\mathcal{A}$ in $\mathcal{B}(n, \alpha)$, an appropriate normalization of the random variable $\log \operatorname{lcm}(\mathcal{A})$ converges in distribution to a standard normal random variable, as $n \rightarrow+\infty$. In light of Theorems 1.2 and 1.3, it is then natural to ask whether the random variable

$$
\frac{\operatorname{deg} \operatorname{lcm}\left([\mathcal{A}]_{q}\right)-\frac{3}{\pi^{2}} \cdot \frac{\alpha \operatorname{Li}_{2}(1-\alpha)}{1-\alpha} \cdot n^{2}}{\sqrt{\mathrm{v}(\alpha) n^{3}}}
$$

converges in distribution to a normal random variable, or to some other random variable.

Another problem could be considering polynomial values, similarly to the results done in the context of integers, and studying $\operatorname{lcm}\left([f(1)]_{q}, \cdots,[f(n)]_{q}\right)$ for $f \in \mathbb{Z}[x]$ or, more generally, $\operatorname{lcm}\left([f(k)]_{q}: k \in \mathcal{A}\right)$ with $\mathcal{A}$ a random set in $\mathcal{B}(n, \alpha)$.

\section{Notation}

We employ the Landau-Bachmann "Big Oh" and "little oh" notations $O$ and $o$, as well as the associated Vinogradov symbol «, with their usual meanings. Any dependence of the implied constants is explicitly stated or indicated with subscripts. For real random variables $X$ and $Y$, depending on some parameters, we say that " $X \sim Y$ with probability $1-o(1)$ ", as the parameters tend to some limit, if for every $\varepsilon>0$ we have $\mathbb{P}[|X-Y|>$ $\varepsilon|Y|]=o_{\varepsilon}(1)$, as the parameters tend to the limit. We let $(a, b)$ and $[a, b]$ denote the greatest common divisor and the least common multiple, respectively, of two integers $a$ and $b$. As usual, we write $\varphi(n), \mu(n), \tau(n)$, and $\sigma(n)$, for the Euler totient function, the Möbius function, the number of divisors, and the sum of divisors, of a positive integer $n$, respectively.

\section{Preliminaries}

In this section we collect some preliminary results needed in later arguments. 
Lemma 3.1 We have

$$
\sum_{m \leq x} \tau(m) \ll x \log x
$$

for every $x \geq 2$.

Proof See, e.g., [20, Part I, Theorem 3.2].

Lemma 3.2 We have

$$
\sum_{\left[e_{1}, e_{2}\right]>x} \frac{1}{e_{1} e_{2}\left[e_{1}, e_{2}\right]} \ll \frac{\log x}{x}
$$

for every $x \geq 2$.

Proof From Lemma 3.1 and partial summation, it follows that

$$
\begin{aligned}
\sum_{m>x} \frac{\tau(m)}{m^{2}} & =\left[\frac{\sum_{m \leq t} \tau(m)}{t^{2}}\right]_{t=x}^{+\infty}+2 \int_{x}^{+\infty} \frac{\sum_{m \leq t} \tau(m)}{t^{3}} \mathrm{~d} t \\
& \ll \int_{x}^{+\infty} \frac{\log t}{t^{2}} \mathrm{~d} t=\left[-\frac{\log t+1}{t}\right]_{t=x}^{+\infty} \ll \frac{\log x}{x}
\end{aligned}
$$

Let $e:=\left(e_{1}, e_{2}\right)$ and $e_{i}^{\prime}:=e_{i} / e$ for $i=1,2$. Then we have

$$
\begin{aligned}
\sum_{\left[e_{1}, e_{2}\right]>x} \frac{1}{e_{1} e_{2}\left[e_{1}, e_{2}\right]} & \leq \sum_{e \geq 1} \frac{1}{e^{3}} \sum_{e_{1}^{\prime} e_{2}^{\prime}>x / e} \frac{1}{\left(e_{1}^{\prime} e_{2}^{\prime}\right)^{2}}=\sum_{e \geq 1} \frac{1}{e^{3}} \sum_{m>x / e} \frac{\tau(m)}{m^{2}} \\
& \ll \sum_{e \leq x / 2} \frac{1}{e^{3}} \frac{\log (x / e)}{x / e}+\sum_{e>x / 2} \frac{1}{e^{3}} \ll \frac{\log x}{x}+\frac{1}{x^{2}} \ll \frac{\log x}{x},
\end{aligned}
$$

as desired.

Let us define

$$
\Phi(x):=\sum_{n \leq x} \varphi(n) \quad \text { and } \quad \Phi\left(a_{1}, a_{2} ; x\right):=\sum_{n \leq x} \varphi\left(a_{1} n\right) \varphi\left(a_{2} n\right),
$$

for every $x \geq 1$ and for all positive integers $a_{1}, a_{2}$.

Lemma 3.3 We have

$$
\Phi(x)=\frac{3}{\pi^{2}} x^{2}+O(x \log x),
$$

for every $x \geq 2$.

Proof See, e.g., [20, Part I, Theorem 3.4].

Lemma 3.4 We have

$$
\Phi\left(a_{1}, a_{2} ; x\right)=C_{1}\left(a_{1}, a_{2}\right) x^{3}+O\left(\sigma\left(a_{1}\right) \sigma\left(a_{2}\right) x^{2}(\log x)^{2}\right),
$$

for every $x \geq 2$, where

$$
C_{1}\left(a_{1}, a_{2}\right):=\frac{a_{1} a_{2}}{3} \sum_{d_{1} d_{2} \geq 1} \frac{\mu\left(d_{1}\right) \mu\left(d_{2}\right)}{d_{1} d_{2}\left[d_{1} /\left(a_{1}, d_{1}\right), d_{2} /\left(a_{2}, d_{2}\right)\right]}
$$

and the series is absolutely convergent. 
Proof From the identity $\varphi(n) / n=\sum_{d \mid n} \mu(d) / d$, it follows that

$$
\begin{aligned}
\sum_{n \leq x} \frac{\varphi\left(a_{1} n\right)}{a_{1} n} \frac{\varphi\left(a_{2} n\right)}{a_{2} n} & =\sum_{n \leq x}\left(\sum_{d_{1} \mid a_{1} n} \frac{\mu\left(d_{1}\right)}{d_{1}} \sum_{d_{2} \mid a_{2} n} \frac{\mu\left(d_{2}\right)}{d_{2}}\right) \\
& =\sum_{\substack{d_{1} \leq a_{1} x \\
d_{2} \leq a_{2} x}} \frac{\mu\left(d_{1}\right)}{d_{1}} \frac{\mu\left(d_{2}\right)}{d_{2}} \#\left\{n \leq x: d_{1} \mid a_{1} n \text { and } d_{2} \mid a_{2} n\right\} \\
& =\sum_{\left[\frac{d_{1}}{\left(a_{1}, d_{1}\right.}, \frac{d_{2}}{\left(a_{2}, d_{2}\right)}\right] \leq x} \frac{\mu\left(d_{1}\right)}{d_{1}} \frac{\mu\left(d_{2}\right)}{d_{2}}\left(\frac{x}{\left[d_{1} /\left(a_{1}, d_{1}\right), d_{2} /\left(a_{2}, d_{2}\right)\right]}+O(1)\right) .
\end{aligned}
$$

Let $c_{i}:=\left(a_{i}, d_{i}\right)$ and $e_{i}:=d_{i} / c_{i}$, for $i=1,2$. On the one hand, we have

$$
E_{1}:=\sum_{\left[\frac{d_{1}}{\left(a_{1}, d_{1}\right)}, \frac{d_{2}}{\left(a_{2}, d_{2}\right)}\right] \leq x} \frac{1}{d_{1} d_{2}} \leq \sum_{c_{1} \mid a_{1}} \frac{1}{c_{1}} \sum_{c_{2} \mid a_{2}} \frac{1}{c_{2}} \sum_{e_{1} \leq x} \frac{1}{e_{1}} \sum_{e_{2} \leq x} \frac{1}{e_{2}} \ll \frac{\sigma\left(a_{1}\right) \sigma\left(a_{2}\right)}{a_{1} a_{2}}(\log x)^{2} .
$$

On the other hand, thanks to Lemma 3.2, we have

$$
\begin{aligned}
E_{2} & :=\sum_{\left[\frac{d_{1}}{\left(a_{1}, d_{1}\right)}, \frac{d_{2}}{\left(a_{2}, d_{2}\right)}\right]>x} \frac{1}{d_{1} d_{2}\left[d_{1} /\left(a_{1}, d_{1}\right), d_{2} /\left(a_{2}, d_{2}\right)\right]} \\
& \leq \sum_{c_{1} \mid a_{1}} \frac{1}{c_{1}} \sum_{c_{2} \mid a_{2}} \frac{1}{c_{2}} \sum_{\left[e_{1}, e_{2}\right]>x} \frac{1}{e_{1} e_{2}\left[e_{1}, e_{2}\right]} \ll \frac{\sigma\left(a_{1}\right) \sigma\left(a_{2}\right)}{a_{1} a_{2}} \frac{\log x}{x},
\end{aligned}
$$

which, in particular, implies that the series

$$
C_{0}\left(a_{1}, a_{2}\right):=\sum_{d_{\mathrm{b}}, d_{2} \geq 1} \frac{\mu\left(d_{1}\right) \mu\left(d_{2}\right)}{d_{1} d_{2}\left[d_{1} /\left(a_{1}, d_{1}\right), d_{2} /\left(a_{2}, d_{2}\right)\right]}
$$

is absolutely convergent. Therefore, we obtain

$$
\begin{aligned}
\sum_{n \leq x} \frac{\varphi\left(a_{1} n\right)}{a_{1} n} \frac{\varphi\left(a_{2} n\right)}{a_{2} n} & =\left(C_{0}\left(a_{1}, a_{2}\right)+O\left(E_{2}\right)\right) x+O\left(E_{1}\right) \\
& =C_{0}\left(a_{1}, a_{2}\right) x+O\left(\frac{\sigma\left(a_{1}\right) \sigma\left(a_{2}\right)}{a_{1} a_{2}}(\log x)^{2}\right)
\end{aligned}
$$

Now (5) follows from (7) by partial summation and since $C_{1}\left(a_{1}, a_{2}\right)=\frac{a_{1} a_{2}}{3} C_{0}\left(a_{1}, a_{2}\right)$.

Remark 3.1 The obvious bound $\varphi(m) \leq m$ yields $C_{1}\left(a_{1}, a_{2}\right) \leq \frac{a_{1} a_{2}}{3}$ (which is not so obvious from (6)).

We end this section with an easy observation that will be useful later.

Remark 3.2 It holds $1-(1-x)^{k} \leq k x$, for all $x \in[0,1]$ and for all integers $k \geq 0$.

\section{Proofs}

Henceforth, let $\mathcal{A}$ be a random set in $\mathcal{B}(n, \alpha)$, let $[\mathcal{A}]_{q}$ be its $q$-analog, and put $L:=$ $\operatorname{lcm}\left([\mathcal{A}]_{q}\right)$ and $X:=\operatorname{deg} L$. For every positive integer $d$, let us define

$$
I_{\mathcal{A}}(d):= \begin{cases}1 & \text { if } d \mid k \text { for some } k \in \mathcal{A} \\ 0 & \text { otherwise }\end{cases}
$$


The following lemma gives a formula for $X$ in terms of $I_{\mathcal{A}}$ and the Euler function.

Lemma 4.1 We have

$$
X=\sum_{1<d \leq n} \varphi(d) I_{\mathcal{A}}(d)
$$

Proof For every positive integer $k$, it holds

$$
[k]_{q}=\frac{q^{k}-1}{q-1}=\prod_{\substack{d \mid k \\ d>1}} \Phi_{d}(q)
$$

where $\Phi_{d}(q)$ is the $d$ th cyclotomic polynomials. Since, as it is well known, every cyclotomic polynomial is irreducible over $\mathbb{Q}$, it follows that $L$ is the product of the polynomials $\Phi_{d}(q)$ such that $d>1$ and $d \mid k$ for some $k \in \mathcal{A}$. Finally, the equality $\operatorname{deg}\left(\Phi_{d}(q)\right)=\varphi(d)$ and the definition of $I_{\mathcal{A}}$ yield (8).

Let $\beta:=1-\alpha$. The next lemma provides two expected values involving $I_{\mathcal{A}}$.

Lemma 4.2 For all positive integers $d, d_{1}, d_{2}$, we have

$$
\mathbb{E}\left[I_{\mathcal{A}}(d)\right]=1-\beta^{\lfloor n / d\rfloor}
$$

and

$$
\mathbb{E}\left[I_{\mathcal{A}}\left(d_{1}\right) I_{\mathcal{A}}\left(d_{2}\right)\right]=1-\beta^{\left\lfloor n / d_{1}\right\rfloor}-\beta^{\left\lfloor n / d_{2}\right\rfloor}+\beta^{\left\lfloor n / d_{1}\right\rfloor+\left\lfloor n / d_{2}\right\rfloor-\left\lfloor n /\left[d_{1}, d_{2}\right]\right\rfloor} .
$$

Proof On the one hand, by the definition of $I_{\mathcal{A}}$, we have

$$
\mathbb{E}\left[I_{\mathcal{A}}(d)\right]=\mathbb{P}[\exists k \in \mathcal{A}: d \mid k]=1-\mathbb{P}\left[\bigwedge_{m \leq\lfloor n / d\rfloor}(d m \notin \mathcal{A})\right]=1-\beta^{\lfloor n / d\rfloor},
$$

which is (9). On the other hand, by linearity of the expectation and by (9), we have

$$
\begin{aligned}
\mathbb{E}\left[I_{\mathcal{A}}\left(d_{1}\right) I_{\mathcal{A}}\left(d_{2}\right)\right] & =\mathbb{E}\left[I_{\mathcal{A}}\left(d_{1}\right)+I_{\mathcal{A}}\left(d_{2}\right)-1+\left(1-I_{\mathcal{A}}\left(d_{1}\right)\right)\left(1-I_{\mathcal{A}}\left(d_{2}\right)\right)\right] \\
& =\mathbb{E}\left[I_{\mathcal{A}}\left(d_{1}\right)\right]+\mathbb{E}\left[I_{\mathcal{A}}\left(d_{2}\right)\right]-1+\mathbb{E}\left[\left(1-I_{\mathcal{A}}\left(d_{1}\right)\right)\left(1-I_{\mathcal{A}}\left(d_{2}\right)\right)\right] \\
& =1-\beta^{\left\lfloor n / d_{1}\right\rfloor}-\beta^{\left\lfloor n / d_{2}\right\rfloor}+\mathbb{E}\left[\left(1-I_{\mathcal{A}}\left(d_{1}\right)\right)\left(1-I_{\mathcal{A}}\left(d_{2}\right)\right)\right],
\end{aligned}
$$

where the last expected value can be computed as

$$
\begin{aligned}
\mathbb{E}\left[\left(1-I_{\mathcal{A}}\left(d_{1}\right)\right)\left(1-I_{\mathcal{A}}\left(d_{2}\right)\right)\right] & =\mathbb{P}\left[\forall k \in \mathcal{A}: d_{1} \nmid k \text { and } d_{2} \nmid k\right] \\
& =\mathbb{P}\left[\bigwedge_{\substack{k \leq n \\
d_{1} \mid k \text { or } d_{2} \mid k}}(k \notin \mathcal{A})\right]=\beta^{\left\lfloor n / d_{1}\right\rfloor+\left\lfloor n / d_{2}\right\rfloor-\left\lfloor n /\left\lfloor d_{1} d_{2}\right]\right\rfloor,}
\end{aligned}
$$

and second claim follows.

We are ready to compute the expected value of $X$. 
Proof of Theorem 1.2 From Lemmas 4.1 and 4.2, it follows that

$$
\mathbb{E}[X]=\sum_{1<d \leq n} \varphi(d) \mathbb{E}\left[I_{\mathcal{A}}(d)\right]=\sum_{1<d \leq n} \varphi(d)\left(1-\beta^{\lfloor n / d\rfloor}\right) .
$$

Moreover, since $\lfloor n / d\rfloor=j$ if and only if $n /(j+1)<d \leq n / j$, we get that

$$
\begin{aligned}
\sum_{d \leq n} \varphi(d)\left(1-\beta^{\lfloor n / d\rfloor}\right) & =\sum_{j \leq n}\left(1-\beta^{j}\right) \sum_{n /(j+1)<d \leq n / j} \varphi(d) \\
& =\sum_{j \leq n}\left(1-\beta^{j}\right)\left(\Phi\left(\frac{n}{j}\right)-\Phi\left(\frac{n}{j+1}\right)\right) \\
& =\alpha \sum_{j \leq n} \beta^{j-1} \Phi\left(\frac{n}{j}\right) \\
& =\frac{3}{\pi^{2}} \cdot \alpha \sum_{j \leq n} \frac{\beta^{j-1}}{j^{2}} \cdot n^{2}+O\left(\alpha \sum_{j \leq n} \frac{n}{j} \log \left(\frac{n}{j}\right)\right) \\
& =\frac{3}{\pi^{2}} \cdot \frac{\alpha \operatorname{Li}_{2}(1-\alpha)}{1-\alpha} \cdot n^{2}+O\left(\alpha n(\log n)^{2}\right),
\end{aligned}
$$

where we used Lemma 3.3. Putting together (10) and (11), and noting that, by Remark 3.2, the addend of (11) corresponding to $d=1$ is $1-\beta^{n}=O(\alpha n)$, we get (2). The proof is complete.

Now we consider the variance of $X$.

Proof of Theorem 1.3 From Lemmas 4.1 and 4.2, it follows that

$$
\begin{aligned}
\mathbb{V}[X] & =\mathbb{E}\left[X^{2}\right]-\mathbb{E}[X]^{2} \\
& =\sum_{1<d_{\mathrm{b}} d_{2} \leq n} \varphi\left(d_{1}\right) \varphi\left(d_{2}\right)\left(\mathbb{E}\left[I_{\mathcal{A}}\left(d_{1}\right) I_{\mathcal{A}}\left(d_{2}\right)\right]-\mathbb{E}\left[I_{\mathcal{A}}\left(d_{1}\right)\right] \mathbb{E}\left[I_{\mathcal{A}}\left(d_{2}\right)\right]\right) \\
& =\sum_{1<d_{\mathrm{b}} d_{2} \leq n} \varphi\left(d_{1}\right) \varphi\left(d_{2}\right) \beta^{\left\lfloor n / d_{1}\right\rfloor+\left\lfloor n / d_{2}\right\rfloor-\left\lfloor n /\left[d_{1}, d_{2}\right]\right\rfloor}\left(1-\beta^{\left\lfloor n /\left[d_{1}, d_{2}\right]\right\rfloor}\right) .
\end{aligned}
$$

Let us define

$$
V_{n}(\alpha):=\frac{1}{n^{3}} \sum_{d_{\mathrm{b}}, d_{2} \leq n} \varphi\left(d_{1}\right) \varphi\left(d_{2}\right) \beta^{\left\lfloor n / d_{1}\right\rfloor+\left\lfloor n / d_{2}\right\rfloor-\left\lfloor n /\left[d_{1}, d_{2}\right]\right\rfloor}\left(1-\beta^{\left\lfloor n /\left[d_{1}, d_{2}\right]\right\rfloor}\right) .
$$

Clearly, we have

$$
V_{n}(\alpha)-\frac{\mathbb{V}[X]}{n^{3}} \ll \frac{1}{n^{3}} \sum_{d \leq n} \varphi(d) \beta^{n}\left(1-\beta^{\lfloor n / d\rfloor}\right) \leq \frac{1}{n^{3}} \sum_{d \leq n} d \ll \frac{1}{n} .
$$

Hence, in order to prove (3), it suffices to show that $V_{n}(\alpha)=\mathrm{v}(\alpha)+o(1)$.

For all vectors $\boldsymbol{a}:=\left(a_{1}, a_{2}\right)$ and $\boldsymbol{j}:=\left(j_{1}, j_{2}, j_{3}\right)$ with components in the set of positive integers, define the quantities

$$
\rho_{1}(\boldsymbol{a}, \boldsymbol{j}):=\max \left(\frac{1}{a_{1}\left(j_{1}+1\right)}, \frac{1}{a_{2}\left(j_{2}+1\right)}, \frac{1}{a_{1} a_{2}\left(j_{3}+1\right)}\right)
$$

and

$$
\rho_{2}(\boldsymbol{a}, \boldsymbol{j}):=\min \left(\frac{1}{a_{1} j_{1}}, \frac{1}{a_{2} j_{2}}, \frac{1}{a_{1} a_{2} j_{3}}\right) .
$$


Let $d:=\left(d_{1}, d_{2}\right)$ and $a_{i}:=d_{i} / d$ for $i=1,2$. Then the equalities

$$
j_{1}=\left\lfloor\frac{n}{d_{1}}\right\rfloor, \quad j_{2}=\left\lfloor\frac{n}{d_{2}}\right\rfloor, \quad j_{3}=\left\lfloor\frac{n}{\left[d_{1}, d_{2}\right]}\right\rfloor,
$$

are equivalent to

$$
j_{1} \leq \frac{n}{a_{1} d}<j_{1}+1, \quad j_{2} \leq \frac{n}{a_{2} d}<j_{2}+1, \quad j_{3} \leq \frac{n}{a_{1} a_{2} d}<j_{3}+1,
$$

which in turn are equivalent to

$$
\frac{n}{a_{1}\left(j_{1}+1\right)}<d \leq \frac{n}{a_{1} j_{1}}, \quad \frac{n}{a_{2}\left(j_{2}+1\right)}<d \leq \frac{n}{a_{2} j_{2}}, \quad \frac{n}{a_{1} a_{2}\left(j_{3}+1\right)}<d \leq \frac{n}{a_{1} a_{2} j_{3}},
$$

that is,

$$
\rho_{1}(\boldsymbol{a}, \boldsymbol{j}) n<d \leq \rho_{2}(\boldsymbol{a}, \boldsymbol{j}) n \text {. }
$$

Therefore, letting

$$
\mathcal{S}_{n}:=\left\{(\boldsymbol{a}, \boldsymbol{j}) \in \mathbb{N}^{5}:\left(a_{1}, a_{2}\right)=1, \exists d \in \mathbb{N} \text { s.t. } \rho_{1}(\boldsymbol{a}, \boldsymbol{j}) n<d \leq \rho_{2}(\boldsymbol{a}, \boldsymbol{j}) n\right\}
$$

and

$$
S(\boldsymbol{a}, \boldsymbol{j} ; n):=\frac{1}{n^{3}} \sum_{\rho_{1}(\boldsymbol{a}, \boldsymbol{j}) n<d \leq \rho_{2}(\boldsymbol{a}, \boldsymbol{j}) n} \varphi\left(a_{1} d\right) \varphi\left(a_{2} d\right),
$$

we have

$$
V_{n}(\alpha)=\sum_{(\boldsymbol{a}, \boldsymbol{j}) \in \mathcal{S}_{n}} \beta^{j_{1}+j_{2}-j_{3}}\left(1-\beta^{j_{3}}\right) S(\boldsymbol{a}, \boldsymbol{j} ; n) .
$$

Now let us define

$$
\mathrm{v}(\alpha):=\sum_{(\boldsymbol{a}, \boldsymbol{j}) \in \mathcal{S}_{\infty}} \beta^{j_{1}+j_{2}-j_{3}}\left(1-\beta^{j_{3}}\right) D(\boldsymbol{a}, \boldsymbol{j}),
$$

where

$$
\mathcal{S}_{\infty}:=\bigcup_{m \geq 1} \mathcal{S}_{m}=\left\{(\boldsymbol{a}, \boldsymbol{j}) \in \mathbb{N}^{5}:\left(a_{1}, a_{2}\right)=1, \rho_{1}(\boldsymbol{a}, \boldsymbol{j})<\rho_{2}(\boldsymbol{a}, \boldsymbol{j})\right\}
$$

and

$$
D(\boldsymbol{a}, \boldsymbol{j}):=C_{1}\left(a_{1}, a_{2}\right)\left(\rho_{2}(\boldsymbol{a}, \boldsymbol{j})^{3}-\rho_{1}(\boldsymbol{a}, \boldsymbol{j})^{3}\right),
$$

recalling that $C_{1}\left(a_{1}, a_{2}\right)$ is defined by (6). The convergence of series (13) follows easily from Remark 3.1, $\rho_{2}(\boldsymbol{a}, \boldsymbol{j}) \leq 1 /\left(a_{1} a_{2} j_{3}\right)$, and the fact that $\min \left(j_{1}, j_{2}\right) \geq j_{3}$ for all $(\boldsymbol{a}, \boldsymbol{j}) \in \mathcal{S}_{\infty}$.

Thanks to Lemma 3.4, for each $(\boldsymbol{a}, \boldsymbol{j}) \in \mathcal{S}_{n}$ we have

$$
S(\boldsymbol{a}, \boldsymbol{j} ; n)=D(\boldsymbol{a}, \boldsymbol{j})+O\left(\sigma\left(a_{1}\right) \sigma\left(a_{2}\right) \rho_{2}(\boldsymbol{a}, \boldsymbol{j})^{2} \cdot \frac{(\log n)^{2}}{n}\right) .
$$

Consequently, we get that

$$
V_{n}(\alpha)=\mathrm{v}(\alpha)-\Sigma_{1}+O\left(\Sigma_{2} \cdot \frac{(\log n)^{2}}{n}\right),
$$

where

$$
\Sigma_{1}:=\sum_{(\boldsymbol{a}, \boldsymbol{j}) \in \mathcal{S}_{\infty} \backslash \mathcal{S}_{n}} \beta^{j_{1}+j_{2}-j_{3}}\left(1-\beta^{j_{3}}\right) D(\boldsymbol{a}, \boldsymbol{j})
$$


and

$$
\Sigma_{2}:=\sum_{(\boldsymbol{a}, \boldsymbol{j}) \in \mathcal{S}_{n}} \beta^{j_{1}+j_{2}-j_{3}}\left(1-\beta^{j_{3}}\right) \sigma\left(a_{1}\right) \sigma\left(a_{2}\right) \rho_{2}(\boldsymbol{a}, \boldsymbol{j})^{2} .
$$

Now we have to bound both $\Sigma_{1}$ and $\Sigma_{2}$.

If $(\boldsymbol{a}, \boldsymbol{j}) \in \mathcal{S}_{\infty} \backslash \mathcal{S}_{n}$ then $\left(\rho_{2}(\boldsymbol{a}, \boldsymbol{j})-\rho_{1}(\boldsymbol{a}, \boldsymbol{j})\right) n<1$ and consequently, also by Remark 3.1,

$$
\begin{aligned}
D(\boldsymbol{a}, \boldsymbol{j}) \ll a_{1} a_{2}\left(\rho_{2}^{3}-\rho_{1}^{3}\right) & =a_{1} a_{2}\left(\rho_{1}^{2}+\rho_{1} \rho_{2}+\rho_{2}^{2}\right)\left(\rho_{2}-\rho_{1}\right) \ll \frac{a_{1} a_{2} \rho_{2}^{2}}{n} \\
& \leq \frac{1}{a_{1} a_{2} j_{3}^{2} n},
\end{aligned}
$$

where, for brevity, we wrote $\rho_{i}:=\rho_{i}(\boldsymbol{a}, \boldsymbol{j})$ for $i=1,2$.

If $(\boldsymbol{a}, \boldsymbol{j}) \in \mathcal{S}_{\infty}$ then, as we already noticed, $\min \left(j_{1}, j_{2}\right) \geq j_{3}$ and, moreover,

$$
\frac{j_{2}}{j_{3}+1}<a_{1}<\frac{j_{2}+1}{j_{3}} \text { and } \frac{j_{1}}{j_{3}+1}<a_{2}<\frac{j_{1}+1}{j_{3}} \text {. }
$$

Hence, we have

$$
\begin{aligned}
\sum_{(a, j) \in \mathcal{S}_{\infty}} \frac{\beta^{j_{1}+j_{2}-j_{3}}\left(1-\beta^{j_{3}}\right)}{a_{1} a_{2} j_{3}^{2}} & \leq \sum_{j_{3} \geq 1} \frac{1-\beta^{j_{3}}}{j_{3}^{2}} \sum_{j_{1}, j_{2} \geq j_{3}} \beta^{j_{1}+j_{2}-j_{3}} \sum_{\substack{j_{2} /\left(j_{3}+1\right)<a_{1}<\left(j_{2}+1\right) / j_{3} \\
j_{1} /\left(j_{3}+1\right)<a_{2}<\left(j_{1}+1\right) / j_{3}}} \frac{1}{a_{1} a_{2}} \\
& \ll \sum_{j_{3} \geq 1} \frac{1-\beta^{j_{3}}}{j_{3}^{2}} \sum_{j_{1}, j_{2} \geq j_{3}} \beta^{j_{1}+j_{2}-j_{3}}=\frac{1}{\alpha^{2}} \sum_{j \geq 1} \frac{\left(1-\beta^{j}\right) \beta^{j}}{j^{2}} \\
& \leq \frac{1}{\alpha} \sum_{j \leq 1 / \alpha} \frac{1}{j}+\frac{1}{\alpha^{2}} \sum_{j>1 / \alpha} \frac{1}{j^{2}} \ll \frac{\log (1 / \alpha)+1}{\alpha}
\end{aligned}
$$

where we used the inequality $1-\beta^{j} \leq \alpha j$, which follows from Remark 3.2.

On the one hand, from (15) and (16) it follows that

$$
\Sigma_{1} \ll \frac{\log (1 / \alpha)+1}{\alpha n}=o(1),
$$

as $\alpha n /\left((\log n)^{3}(\log \log n)^{2}\right) \rightarrow+\infty$ (actually, $\alpha n / \log n \rightarrow+\infty$ is sufficient).

On the other hand, from $\rho_{2}(\boldsymbol{a}, \boldsymbol{j}) \leq 1 /\left(a_{1} a_{2} j_{3}\right)$, (16), and the bound $\sigma(m) \ll m \log \log m$ (see, e.g., [20, Part I, Theorem 5.7]) it follows that

$$
\begin{aligned}
\Sigma_{2} & \leq \sum_{(\boldsymbol{a}, \boldsymbol{j}) \in \mathcal{S}_{n}} \frac{\beta^{j_{1}+j_{2}-j_{3}}\left(1-\beta^{j_{3}}\right)}{a_{1} a_{2} j_{3}^{2}} \cdot \frac{\sigma\left(a_{1}\right) \sigma\left(a_{2}\right)}{a_{1} a_{2}} \ll \frac{(\log (1 / \alpha)+1)(\log \log n)^{2}}{\alpha} \\
& =o\left(\frac{n}{(\log n)^{2}}\right)
\end{aligned}
$$

as $\alpha n /\left((\log n)^{3}(\log \log n)^{2}\right) \rightarrow+\infty$.

At this point, putting together (14), (17), and (18), we obtain $V_{n}(\alpha)=\mathrm{v}(\alpha)+o(1)$, as desired. The proof of (3) is complete.

It remains only to prove the upper bound (4). From (12) it follows that

$$
\begin{aligned}
\mathbb{V}[X] & \leq \sum_{\left[d_{1}, d_{2}\right] \leq n} \varphi\left(d_{1}\right) \varphi\left(d_{2}\right) \beta^{\left\lfloor n / d_{1}\right\rfloor+\left\lfloor n / d_{2}\right\rfloor-\left\lfloor n /\left[d_{1}, d_{2}\right]\right\rfloor}\left(1-\beta^{\left\lfloor n /\left[d_{1}, d_{2}\right]\right\rfloor}\right) \\
& \leq \sum_{\left[d_{1} d_{2}\right] \leq n} d_{1} d_{2} \cdot \frac{\alpha n}{\left[d_{1}, d_{2}\right]}=\alpha n \sum_{\left[d_{1}, d_{2}\right] \leq n}\left(d_{1}, d_{2}\right) \leq \alpha n \sum_{d \leq n} d \sum_{a_{1} a_{2} \leq n / d} 1 \\
& =\alpha n \sum_{d \leq n} d \sum_{m \leq n / d} \tau(m) \ll \alpha n^{2} \sum_{d \leq n} \log \left(\frac{n}{d}\right)=\alpha n^{2}(n \log n-\log (n !))<\alpha n^{3},
\end{aligned}
$$


where we used Remark 3.2, Lemma 3.1, and the bound $n !>(n / \mathrm{e})^{n}$. Thus (4) is proved.

Proof of Theorem 1.4 By Chebyshev's inequality, Theorems 1.2 and 1.3, we have

$$
\mathbb{P}[|X-\mathbb{E}[X]|>\varepsilon \mathbb{E}[X]] \leq \frac{\mathbb{V}[X]}{(\varepsilon \mathbb{E}[X])^{2}} \ll \frac{\alpha n^{3}}{(\varepsilon \alpha n)^{2}}=\frac{1}{\varepsilon^{2} \alpha n}=o_{\varepsilon}(1),
$$

as $\alpha n \rightarrow+\infty$. Hence, using again Theorem 1.2, we get

$$
X \sim \frac{3}{\pi^{2}} \cdot \frac{\alpha \operatorname{Li}_{2}(1-\alpha)}{1-\alpha} \cdot n^{2}
$$

with probability $1-o(1)$, as $\alpha n \rightarrow+\infty$.

\section{Authors' contributions}

The author thanks the anonymous referee, whose careful reading and detailed suggestions led to a considerable improvement of the paper.

Funding Open access funding provided by Politecnico di Torino within the CRUI-CARE Agreement.

Received: 27 December 2020 Accepted: 2 February 2021 Published online: 18 February 2021

\section{References}

1. Alsmeyer, G., Kabluchko, Z., Marynych, A.: Limit theorems for the least common multiple of a random set of integers. Trans. Am. Math. Soc. 372(7), 4585-4603 (2019)

2. Andrews, G.E.: $q$-Series: Their Development and Application in Analysis, Number Theory, Combinatorics, Physics, and Computer Algebra, CBMS Regional Conference Series in Mathematics, vol. 66, Published for the Conference Board of the Mathematical Sciences, Washington, DC; by the American Mathematical Society, Providence, RI (1986)

3. Bateman, P., Kalb, J., Stenger, A.: Solution to problem 10797: a limit involving least common multiples. Am. Math. Monthly 109(4), 393-394 (2002)

4. Bostan, A., Marynych, A., Raschel, K.: On the least common multiple of several random integers. J. Number Theory 204, 113-133 (2019)

5. Buraczewski, D., Iksanov, A., Marynych, A.: A Brownian weak limit for the least common multiple of a random $m$-tuple of integers. https://arxiv.org/abs/2004.05643

6. Cilleruelo, J.: The least common multiple of a quadratic sequence. Compos. Math. 147(4), 1129-1150 (2011)

7. Cilleruelo, J., Guijarro-Ordóñez, J.: Ratio sets of random sets. Ramanujan J. 43(2), 327-345 (2017)

8. Cilleruelo, J., Ramana, D.S., Ramaré, O.: Quotient and product sets of thin subsets of the positive integers. Proc. Steklov Inst. Math. 296(1), 52-64 (2017)

9. Cilleruelo, J., Rué, J., Šarka, P., Zumalacárregui, A.: The least common multiple of random sets of positive integers. J. Number Theory 144, 92-104 (2014)

10. Hong, S., Qian, G., Tan, Q.: The least common multiple of a sequence of products of linear polynomials. Acta Math. Hungar. 135(1-2), 160-167 (2012)

11. Kac, V., Cheung, P.: Quantum Calculus. Universitext. Springer, New York (2002)

12. Mastrostefano, D.: On maximal product sets of random sets, (to appear on Journal of Number Theory) https://arxiv. org/abs/2005.04663

13. Maynard, J., Rudnick, Z.: A lower bound on the least common multiple of polynomial sequences, (to appear on Rivista di Matematica dell'Università di Parma) https://arxiv.org/abs/1910.13218

14. Rudnick, Z., Zehavi, S.: On Cilleruelo's conjecture for the least common multiple of polynomial sequences, https:// arxiv.org/abs/1902.01102

15. Rué, J., Šarka, P., Zumalacárregui, A.: On the error term of the logarithm of the lcm of a quadratic sequence. J. Théor. Nombres Bordeaux 25(2), 457-470 (2013)

16. Sah, A.: An improved bound on the least common multiple of polynomial sequences. J. Théor. Nombres Bordeaux 32(3), 891-899 (2020)

17. Sanna, C.: On the L.C.M. of shifted Fibonacci numbers https://arxiv.org/abs/2007.13330

18. Sanna, C.: A note on product sets of random sets. Acta Math. Hungar. 162(1), 76-83 (2020)

19. Sanna, C.: On the L.C.M. of random terms of binary recurrence sequences. J. Number Theory $\mathbf{2 1 3}, 221-231$ (2020)

20. Tenenbaum, G.: Introduction to Analytic and Probabilistic Number Theory, third ed., Graduate Studies in Mathematics, vol. 163, American Mathematical Society, Providence, RI, Translated from the 2008 French edition by Patrick D. F. Ion (2015)

\section{Publisher's Note}

Springer Nature remains neutral with regard to jurisdictional claims in published maps and institutional affiliations. 